C. Wyder-Westh • A. Duppenthaler •

M. Gorgievski-Hrisoho • C. Aebi

\title{
Evaluation of Two Rapid Detection Assays for Identification of Respiratory Syncytial Virus in Nasopharyngeal Secretions of Young Children
}

Published online: 8 November 2003

(C) Springer-Verlag 2003

Respiratory syncytial virus (RSV) is the single most important lower respiratory tract pathogen in infants and toddlers [1]. Up to $70 \%$ of the annual birth cohort are infected during the first year of life [2], and 1-2\% are hospitalized annually for RSV bronchiolitis or pneumonia $[3,4]$. Since RSV is highly contagious, hospital admission entails infection control measures for prevention of contact and droplet transmission. Thus, clinicians should have available a rapid test that identifies RSV-infected patients quickly and reliably in the emergency room. The test result enables the implementation of adequate infection control precautions at the time the patient is transferred to the floor and provides a rationale for the cohorting of patients with acute respiratory tract disease. In addition, inadequate use of antibiotics may be avoided. Bedside RSV rapid antigen detection systems have been available for several years. Their usefulness in clinical practice has been limited by relatively poor sensitivity. Recently, two new test systems have become available commercially. The purpose of the present study was to compare these new assays with antigen detection using a direct fluorescence assay (DFA).

During the 2002/2003 RSV peak season, nasopharyngeal secretions (NPS) from 30 children (median age, 5.4 months; range, $0.5-64$ months) referred consecutively to the emergency department of the University of Bern Children's Hospital for suspected RSV infection were evaluated. NPS specimens were sampled transnasally using a Vygon infant mucus aspirator (Vygon, Ecouen, France), were brought to a total volume of $2.0 \mathrm{ml}$ using sterile normal saline, and were kept at $4^{\circ} \mathrm{C}$ for a maximum of $24 \mathrm{~h}$ before processing. Sample surplus

\footnotetext{
C. Wyder-Westh · A. Duppenthaler · C. Aebi $(\square)$

Department of Pediatrics, University of Bern,

Inselspital, 3010 Bern, Switzerland

e-mail: christoph.aebi@insel.ch

Tel.: +41-31-6329487

Fax: +41-31-632-9468
}

M. Gorgievski-Hrisoho · C. Aebi

Institute for Infectious Diseases,

University of Bern, Inselspital, 3010 Bern, Switzerland was stored at $-80^{\circ} \mathrm{C}$. In addition to DFA (Light Diagnostics Respiratory Panel DFA, Chemicon International, USA), which is currently the standard diagnostic procedure used at this institution, aliquots of NPS were tested using (i) the RSV OIA assay (OIA) (Thermo BioStar, USA); (ii) the NOW RSV Test (Binax, USA); (iii) RSV culture using shell vial technology; and, in selected cases, (iv) reverse transcriptase polymerase chain reaction (PCR) (Hexaplex; Prodesse, USA). All tests were carried out according to the manufacturer's instructions. OIA and NOW were performed in the emergency department by one of the authors (C.W.). DFA, culture and PCR were performed by professional diagnostic microbiology personnel. Detection of RSV by culture was considered unequivocal proof of infection. Culture-negative samples testing positive in one of the antigen detection assays were re-tested using PCR as the arbiter. Thus, the gold standard consisted of a combination of culture and PCR.

RSV was detected in 22 of 30 samples. Culture was positive in 20 cases, PCR detected RSV RNA in two additional cases. Overall, antigen test(s) yielded a positive result in six culture-negative samples. PCR was positive in two of these specimens. Table 1 summarizes the performance of the two bedside RSV detection kits, OIA and NOW, in comparison with DFA.

In this small population of young children with acute respiratory tract illness and a high pre-test probability of RSV infection, the three antigen detection kits performed similarly (Table 1) and the results were comparable to studies evaluating other rapid antigen detection systems $[5,6,7,8]$. These test performances are unlikely to be applicable to inter-epidemic periods with low or absent RSV activity and to older individuals in whom viral titers in nasopharyngeal secretions are expected to be lower. While DFA requires specific equipment (i.e., fluorescence microscope) and trained personnel for reading the test results, the test procedures for both OIA and NOW are designed for bedside testing by non-laboratory personnel. Both tests require approximately $15 \mathrm{~min}$ for completion. The NOW test procedure is a single manual step, which 
Table 1 Diagnostic performance of three respiratory syncytial virus (RSV) antigen detection assays

\begin{tabular}{llccc}
\hline Method & Sensitivity (\%) & Specificity (\%) & Positive predictive value (\%) & Negative predictive value $(\%)$ \\
\hline RSV OIA $^{\mathrm{a}}$ & 87.5 & 75.0 & 90.5 & 66.7 \\
RSV NOW $^{\mathrm{b}}$ & 86.4 & 100.0 & 100.0 & 72.7 \\
RSV DFA $^{\mathrm{c}}$ & 95.5 & 100.0 & 100.0 & 88.9 \\
\hline
\end{tabular}

${ }^{a}$ Respiratory Syncytial Virus Optical ImmunoAssay (Thermo BioStar, USA)

b Respiratory Syncytial Virus NOW RSV Test Kit (Binax, USA)

${ }^{c}$ Respiratory Syncytial Virus Direct Fluorescence Assay (Light Diagnostics Respiratory Panel DFA, Chemicon International, USA)

consists of the administration of $100 \mu \mathrm{l}$ of NPS to the test strip using a calibrated pipet, and allows the test performer to walk away during the incubation period. The test surface is easy to read. In contrast, the OIA test procedure is complex, involves the use of three different reagents and a wash solution, and entails three incubation steps. The OIA test is thus error-prone and clearly less suitable for bedside RSV testing by non-laboratory medical personnel. In addition, reading of the test surface requires some experience. Both kits share a relatively low negative predictive value. Until data from larger study populations are available, backup testing using DFA, culture or PCR is mandatory to rule out infection reliably if clinical suspicion of RSV infection persists in a patient tested negative. In summary, these two new tests offer performance characteristics that are comparable to DFA and other rapid antigen detection systems. Logistic and quality control considerations clearly favor the NOW test for bedside use in the pediatric emergency department.

\section{References}

1. Hall CB (2001) Respiratory syncytial virus and parainfluenza virus. New Engl J Med 344:1917-1928

2. Glezen WA, Taber LL, Frank AL, Kasel JA (1996) Risk of primary infection and reinfection with respiratory syncytial virus. Am J Dis Childhood 140:543-546

3. Prober CG (1997) Reducing the morbidity of lower respiratory tract infections caused by respiratory syncytial virus: still no answer. Pediatrics 99:472-475

4. Duppenthaler A, Gorgievski-Hrisoho M, Aebi C (2001) Regional impact of prophylaxis with the monoclonal antibody palivizumab on hospitalisations for respiratory syncytial virus. Swiss Medical Weekly 131:146-151

5. Krilov LR, Lipson SM, Barone SR, Kaplan MH, Ciamician Z, Harkness SH (1994) Evaluation of a rapid diagnostic test for respiratory syncytial virus (RSV): potential for bedside testing. Pediatrics 93:903-906

6. Todd SJ, Minnich L, Waner JL (1995) Comparison of rapid immunofluorescence procedure with TestPack RSV and Directigen FLU-A for diagnosis of respiratory syncytial virus and influenza A virus. J Clin Microbiol 33:1650-1651

7. Olsen MA, Shuck KM, Sambol AR (1993) Evaluation of Abbott TestPack RSV for the diagnosis of respiratory syncytial virus infections. Diagn Microbiol Infect Dis 16:105-109

8. Ribes JA, Seabolt JP, Overman SB (2002) Performance characteristics of VIDAS and Directigen respiratory syncytial virus (RSV) antigen detection assays and culture for the identification of RSV in respiratory specimens. J Clin Microbiol 40:1818-1820 